\title{
La utilización de las TICs en la actividad académica de los estudiantes universitarios con discapacidad
}

\section{The use of ICT in the academic activity of college students with disabilities}

\author{
Carmen ALBA PASTOR y Ainara ZUBILLAGA DEL RÍO \\ Universidad Complutense de Madrid y Universidad Camilo José Cela
}

Recibido: Marzo 2012

Aceptado: Abril 2012

\begin{abstract}
Resumen
Existe un creciente interés en las instituciones universitarias por responder a las necesidades de los estudiantes con discapacidad. Varios son los elementos vinculados a su participación académica y forman un conjunto de factores que, según como se articulen, pueden constituir una barrera o un apoyo en el proceso de inclusión. La tecnología es uno de ellos, y esta dualidad el punto de partida del presente trabajo que está dirigido a identificar el rol que juegan las TIC en el desarrollo de la vida académica de los estudiantes con discapacidad en la universidad, las tecnologías que utilizan, el uso de la web y el campus virtual y su accesibilidad, para su utilización, con el fin de determinar en qué medida y bajo qué circunstancias constituyen una barrera o un apoyo para dicho colectivo.
\end{abstract}

Palabras clave: Discapacidad, Educación Superior, Tecnología, Accesibilidad

\begin{abstract}
There is growing interest in Higher Education institutions to respond to the needs of students with disabilities. There are several elements linked to their academic participation which conform a set of factors that could act as a barrier or as a support in the inclusion process. Technology is one of them. Its duality is in the origin of this work, aimed to identify the role played by ICT in the development of academic life of students with disabilities in higher education settings, in reference to the technologies they use and the accessibility of the university webpage and the learning platform ("virtual campus"), to establish what and under which circumstances technology is a barrier or a support for this group of students.
\end{abstract}

Keywords: Disability, Higher Education, Technology, Accessibility

\section{La atención a la diversidad como necesidad social}

La atención a la discapacidad constituye uno de los compromisos actuales más emergentes, y se ha convertido en punto de interés tanto a nivel público como privado. En los últimos treinta años hemos observado un cambio sustancial en el carácter inclusivo de la sociedad, evolucionando en la toma de conciencia de la diversidad como elemento constitutivo básico y con ello, en la conceptualización e incorporación 
de la discapacidad como parte de esta diversidad inherente. De igual forma, también ha cambiado la atención y las medidas puestas en marcha para lograr la igualdad de derechos efectiva de este colectivo en los diferentes ámbitos de la sociedad, siendo la educación uno de los sectores clave para ello.

En el ámbito educativo la discapacidad también se está alzando como uno de los focos clave de estudios e investigaciones, de los cuales han resultado propuestas de cada vez mayor arraigo como la "Educación Inclusiva" o la "Educación para Todos". La escolarización generalizada en el sistema educativo ordinario de los niños y niñas con necesidades educativas especiales es sin lugar a dudas una de las claves que ha permitido a estos estudiantes realizar los estudios que les han abierto el acceso a la universidad y demostrar el limitado carácter limitante de muchas discapacidades.

Dentro del campo de la educación, la temática se singulariza si la cuestión se enmarca en el área de la Educación Superior. La naturaleza y peculiaridades de esta etapa del sistema educativo exigen una mayor atención a aquellas variables que intervienen en la atención a la discapacidad, y exige una revisión de los principios inclusivos para adaptarlos a las características de esta etapa educativa. Estas particularidades constituyen el punto de partida para la introducción de conceptos como el Diseño Universal en los principios de la práctica docente universitaria (Burgstahler, 2011).

Los datos ponen de manifiesto una escasa presencia de estudiantes con discapacidad (EcD) en las aulas universitarias. La última Encuesta sobre Discapacidad, Autonomía Personal y Situaciones de Dependencia (INE, 2008) evidencia que existe un bajo nivel formativo entre el colectivo de personas con discapacidad: frente a un $29,30 \%$ de la población con discapacidad que finaliza sus estudios primarios, esta cifra se reduce de manera significativa a un $5,26 \%$ en el caso de estudios universitarios. Estas cifras revelan que el contexto universitario encuentra en la atención a la diversidad una asignatura pendiente que, sin embargo, en la mayoría de las instituciones universitarias, ya están intentando resolver.

Sobre el creciente interés de las instituciones universitarias por responder a las necesidades de los estudiantes con discapacidad, eliminar barreras y prevenir la discriminación negativa, Barfield, Bennett, Folio y Killman (2007) señalan entre las acciones que se están llevando a cabo de manera más sistemática, el establecimiento del requisito de acreditar la discapacidad, la solicitud del servicio iniciada por el estudiante con discapacidad, medidas para mejorar la accesibilidad, los ajustes académicos, y, algo todavía poco extendido en nuestro contexto, como es el establecimiento de un procedimiento de reclamación para el estudiante. También se cuenta, cuando es posible, con la información que puede aportar el estudiante o el centro del que procede sobre los ajustes que ha venido recibiendo en los niveles anteriores del sistema educativo o los que son necesarios para su incorporación plena a la vida universitaria. 


\section{El impulso a la accesibilidad de las universidades en el marco normativo}

Garantizar la accesibilidad de las universidades a través de la puesta en marcha de acciones que permitan a las personas con discapacidad desarrollar su actividad profesional, académica y social en estas instituciones es, también, una obligación derivada de la normativa vigente. De carácter general, y basada en el principio constitucional del derecho de todos a la educación (Artículo $21 \mathrm{CE}$ 1978) y la responsabilidad de los poderes públicos de realizar "una política de previsión, tratamiento, rehabilitación e integración de los disminuidos físicos, sensoriales y psíquicos, a los que prestará la atención especializada que requieran y los ampararán especialmente para el disfrute de los derechos" (Artículo 49 CE 1978), la Ley Orgánica 6/2001, de 21 de diciembre, de Universidades (LOU) establece en su Artículo 46 que los estudiantes tendrán derecho a "la igualdad de oportunidades y no discriminación, por circunstancias personales o sociales, incluida la discapacidad, en el acceso a la Universidad, ingreso en los centros, permanencia en la Universidad y ejercicio de sus derechos académicos".

Posteriormente, se han introducido modificaciones a la LOU, entre otras, dirigidas hacia el "impulso de políticas activas para garantizar la igualdad de oportunidades a las personas con discapacidad". La Ley Orgánica 4/2007, de 12 de abril, por la que se modifica la Ley Orgánica 6/2001, de 21 de diciembre, de Universidades, supone una mejora sustancial en cuanto a la presencia de las personas con discapacidad en el desarrollo normativo universitario. Dentro de estas modificaciones, hay que destacar la Disposición adicional vigésimo cuarta. De la inclusión de las personas con discapacidad en la universidades, que recoge seis líneas de actuación básica:

a) El establecimiento de "medidas de acción positiva" orientadas a garantizar la participación de toda la comunidad universitaria ("estudiantes y demás miembros de la comunidad universitaria con discapacidad") en el ámbito de la Educación Superior.

b) La no discriminación en el "acceso, el ingreso, la permanencia y el ejercicio de los títulos académicos y de otra clase que tengan reconocidos".

c) El impulso de acciones dirigidas a que "todos los miembros de la comunidad universitaria que presenten necesidades especiales o particulares asociadas a la discapacidad dispongan de los medios, apoyos y recursos que aseguren la igualdad real y efectiva de oportunidades".

d) La necesidad de que "edificios, instalaciones y dependencias de las universidades, incluidos también los espacios virtuales, así como los servicios, procedimientos y el suministro de información" sean accesibles para todos.

e) La promoción de contenidos relacionados con los Derechos Humanos, accesibilidad, universidad y Diseño para Todos en los planes de estudio.

f) El derecho de los estudiantes con discapacidad a "la exención total de tasas y precios públicos en los estudios conducentes a la obtención de un título universitario". 
Concurrente en el objetivo de la accesibilidad, la Ley 51/2003, de 2 de diciembre, de igualdad de oportunidades, no discriminación y accesibilidad universal de las personas con discapacidad, de acuerdo a su principio de transversalidad, es aplicable a diferentes condiciones ambientales tales como las telecomunicaciones, infraestructuras y edificación, transportes, bienes y servicios a disposición del público y relaciones con las Administraciones Públicas.

Dos son los aspectos fundamentales que establece y regula esta ley. Por un lado, por primera vez un texto normativo contempla el concepto de "exigencias de accesibilidad". Y no se habla de medidas, actuaciones o planes dirigidos a la consecución de entornos accesibles, sino de "requisitos que deben cumplir los entornos, productos y servicios, así como las condiciones de no discriminación en normas, criterios y prácticas, con arreglo a los principios de accesibilidad universal de diseño para todos" (Artículo 7). Es decir, que en su aplicación al ámbito universitario, los entornos de aprendizaje, productos y servicios universitarios deben cumplir los principios de accesibilidad universal que permitan el acceso y participación de todos los estudiantes, y, por lo tanto, responder a las necesidades de los estudiantes con discapacidad.

Y, por otro, se contempla la provisión de "apoyos complementarios, tales como ayudas económicas, tecnológicas de apoyo, servicios o tratamientos especializados y otros servicios personales. En particular, ayudas y servicios auxiliares para la comunicación, como sistemas aumentativos y alternativos, sistemas de apoyos a la comunicación oral y lengua de signos $\mathrm{u}$ otros dispositivos que permitan la comunicación" (Artículo 10.2). Las universidades tienen que asumir la responsabilidad de proporcionar estos apoyos a los estudiantes que lo requieran como elementos que deben formar parte de los entornos de aprendizaje para garantizar la accesibilidad de los mismos a todos los estudiantes, y que no sea, como hasta ahora, "un problema del estudiante" con discapacidad.

El modelo actual de discapacidad aboga porque los problemas y desventajas a las que ha de enfrentarse una persona con discapacidad, no sólo tiene su origen en las dificultades inherentes a su discapacidad, sino muy especialmente en el entorno que le rodea. El acceso a las instalaciones, edificios, infraestructuras, productos y servicios que componen, en este caso, la universidad, es un requisito básico para la integración y participación real y efectiva de las personas con discapacidad en la vida universitaria. Así queda recogido, no sólo en las modificaciones introducidas en la LOU (Ley Orgánica 4/2007. Disposición adicional vigésimo cuarta), sino también en toda la normativa referida a la accesibilidad universal. 


\section{Elementos vinculados a la participación académica de las personas con discapacidad en la universidad}

Los trabajos más relevantes acerca de la vida universitaria de los estudiantes con discapacidad coinciden en señalar, como factores clave en su estudio, la existencia de barreras y la emergente presencia de apoyos. En este sentido, destaca el trabajo de Fichten et al. (2005 y 2010), cuyo propósito consistía en averiguar qué factores actúan facilitando o dificultando los estudios universitarios tanto para estudiantes con discapacidad $(n=74)$, como sin discapacidad $(n=154)$, intentando identificarlos. Los resultados mostraron que los apoyos más relevantes para las personas con discapacidad son las adaptaciones y acomodaciones, así como el transporte público accesible. Sin embargo, lo más significativo del estudio es el hallazgo de facilitadores que son comunes a los dos grupos, como son los amigos, tecnología, disponibilidad de materiales del curso, nivel de motivación personal y accesibilidad a las aulas y laboratorios.

En este contexto de barreras y apoyos, una primera línea de trabajo interesante versa sobre aquellas condiciones que se constituyen como barreras para los estudiantes con discapacidad y que no sólo son físicas, sino también actitudinales, didácticas, organizativas y tecnológicas. Trabajos recientes también han ido delimitando esferas de actividad y categorías de relación en las que los estudiantes con discapacidad pueden enfrentarse a dificultades específicas, sin que encuentren siempre respuesta a ello. No se trata tanto de saber dónde pueden aparecer las dificultades, sino qué efectos pueden tener y cómo resolverlas. En último extremo, de lo que se trata es de saber cómo evitar que las instituciones y la vida en la universidad puedan convertirse en algo hostil, y de otro lado, cómo hacer posible que la experiencia en Educación Superior para este colectivo resulte tan enriquecedora y positiva como pueda serlo para el resto.

En relación con este propósito, surge también una serie de trabajos que, más allá de las dificultades, atienden e indagan sobre la satisfacción de los estudiantes con discapacidad respecto a los apoyos recibidos en las instituciones de Enseñanza Superior, así como sobre la eficacia y acierto de los apoyos, técnicos e institucionales puestos a su disposición. Dentro de esta línea de investigación, resulta de especial interés la investigación de West, Kregel, Getzel, Zhu, Ipsen y Martin (1993). Con una muestra de 40 estudiantes, las respuestas generalmente expresaban satisfacción con los servicios recibidos en los centros. No obstante, la mayoría de los casos, también manifestaron haber encontrado barreras en su educación, falta de comprensión y cooperación por parte de la administración, profesores, servicios y compañeros, así como en las medidas de adaptación y de accesibilidad a los edificios y servicios.

Conocer las conclusiones de los estudios sobre las barreras y apoyos presentes en la vida académica de los estudiantes con discapacidad pone de manifiesto que nada es concluyente, sino que existen una serie de elementos clave que, en función de su planteamiento y desarrollo, pueden convertirse en un factor de exclusión o de inclusión en la universidad para este colectivo. En este sentido, cabría analizar cuáles son estos elementos, con el fin de ver en qué medida pueden actuar como barreras y de qué manera pueden convertirse en medios de apoyo. 
Las actitudes serían el primero de dichos elementos, entendidas como un factor central en el desarrollo de la vida universitaria de estudiantes con discapacidad. Dentro de este apartado, adquiere un especial relieve la actitud en el profesorado y, por este motivo, se va a profundizar en este contenido en el siguiente apartado. Existen estudios (Rao, 2002; Williamson, 2000) que intentan analizar la posible correlación entre las actitudes positivas en el profesorado y la obtención de resultados satisfactorios en los estudiantes con discapacidad. A pesar de que estas investigaciones no son concluyentes al respecto, sí que se puede afirmar que la correlación positiva entre una cosa y otra es, de cualquier manera, plausible.

Dentro de lo que se podría denominar factor humano, el colectivo de profesores es el segundo de los elementos implicados. Stodden (2001), al estudiar los altos índices de abandono de las carreras universitarias por parte de los estudiantes con discapacidad, identifica como una de las principales causas la falta de conciencia por parte del profesorado en la atención a este colectivo. Una de las dimensiones más significativas de la relación del profesorado con los estudiantes con discapacidad está referida a sus percepciones y creencias acerca de este tema. Bourke, Strehorn y Silver (2000) consideran que la disposición de los profesores a llevar a cabo las adaptaciones está directamente relacionada con sus creencias sobre la eficacia de las mismas para estos estudiantes. En este sentido, Vasek (2005) afirma que resulta común encontrar actitudes de escepticismo o de abierto rechazo que entienden tales adecuaciones como un privilegio, es decir, un trato de favor injusto para el resto de los estudiantes. En cambio, sí que muestran buena disposición a facilitar el trabajo a este tipo de alumno. Así, se puede decir que las diferencias en la disposición de los profesores tienen que ver con el tipo de adaptación que se requiera. Bourke, Strehorn y Silver (2000) apuntan que se podría considerar una "jerarquía de acomodaciones" en función de la facilidad en su aplicación y la distinta disponibilidad del profesorado para desarrollarlas.

Respecto al tema de las estrategias docentes para la atención a la discapacidad, no existen muchas investigaciones al respecto. Destaca el trabajo de Konur (2006) y Tincani (2004), quienes presentan diferentes estrategias con las que reforzar la posibilidad de éxito académico. Las más frecuentes aluden a adaptación del tipo de examen, adaptación de plazos para entrega de trabajos, ajustes en los temarios, etc. Una de las estrategias a tener en cuenta por su presencia cada vez mayor en la formación, y a la que se está dedicando una especial atención en las investigaciones, son las tecnologías.

\section{Las tecnologías como barrera o apoyo del proceso de participación académica y social en la universidad}

Las tecnologías constituyen un claro reflejo del carácter ambiguo de estos elementos por poder suponer un apoyo o una barrera, en función de la perspectiva desde la que se desarrolle. Las Tecnologías de la Información y la Comunicación (TIC) están cada vez más extendidas en la Educación Superior, especialmente aquellas basadas en Internet y las plataformas virtuales de formación. Sin embargo, estos recursos, tan prometedores inicialmente para superar barreras físicas y que, cargados de grandes expectativas para las personas con discapacidad, con frecuencia han 
supuesto nuevas barreras que dificultan el acceso a los estudios y servicios que ofrecen los centros universitarios, profundizando en ocasiones en la denominada brecha digital.

Las tecnologías de apoyo o ayudas técnicas tienen el potencial de permitir el acceso al curriculum y a las actividades de comunicación para los estudiantes con discapacidad. Ahora bien, dada la amplitud de ayudas existentes y las necesidades específicas de cada persona, es necesario tomar decisiones técnicas con respecto a las opciones de software y hardware más adecuadas, en función de las capacidades de la persona y teniendo en cuenta las diferentes tareas para las que pueden ser necesarias, relacionadas especialmente con el estudio, la realización de actividades de aprendizaje y evaluación, trabajos, gestiones administrativas, investigación y comunicación (Ari y Inan, 2010; Fichten, Asuncion, Nguyen, Budd y Amsel,2010).

Pero la utilización de las tecnologías en los procesos educativos universitarios no sólo es cuestión de establecer un amplio catálogo de ayudas técnicas, sino de lograr que los recursos y servicios estén disponibles y que los desarrollos tecnológicos sean accesibles para cualquier estudiante (Gillette, 2006, Lewis, Yoder, Riley, So y Yusufali, 2007; Zubillaga , 2006; Alba, 2005). Los nuevos servicios electrónicos y el aumento de los contextos, plataformas y materiales de aprendizaje en línea ofrecen, sin duda, nuevas oportunidades, pero con ellas, también aparecen nuevas barreras para la accesibilidad en la educación superior. A pesar del aumento del número de estudiantes con discapacidad en la educación superior, la mayoría de las universidades no han garantizado la accesibilidad plena de los entornos de aprendizaje, ni de los recursos y contenidos de aprendizaje en línea para todos los estudiantes,

Pero si bien son importantes y necesarios los servicios y apoyos específicos, parece que las universidades tienen que utilizar planteamientos más holísticos que busquen no solo la accesibilidad para una persona, un servicio o tecnología, sino el logro de la accesibilidad del entorno globalmente, tal y como se plantea desde el modelo del diseño universal para los entornos educativos y los procesos de enseñanza y aprendizaje, para lo que sin duda pueden ser de gran utilidad las tecnologías de la información y de la comunicación (Rose y Meyer, 2002; Zeff, 2007).

Una de los aspectos a considerar dentro del ámbito de las tecnologías hace referencia a las páginas Web. En este caso, y puesto que el ámbito de estudio está centrado en la Educación Superior, parece lícito evidenciar aquí trabajos orientados a analizar la accesibilidad de las páginas Web de universidades u otros establecimientos de Educación Superior. A este respecto, es preciso nombrar un estudio realizado por Flowers, Bray y Algozzine (2001) sobre 260 páginas Web de universidades en EE.UU., analizadas mediante el programa de revisión automática Bobby 3.2. Estos autores encontraron que más de las tres cuartas partes (77\%) de ellas no cumplían con las pautas de accesibilidad. En el contexto de las universidades españolas, destacan trabajos como los de Toledo (2001), Termens y Ribera (2002), Alba, Zubillaga y Ruíz (2003) y Observatorio de Infoaccesibilidad (2010, 2006), donde se concluye que, si bien existe una tendencia hacia una mayor accesibilidad en las páginas Web de las universidades, aún queda mucho camino por recorrer al respecto. En definitiva, y a pesar de que se están tomando medidas (por ejemplo, la Ley de Igualdad de Oportunidades, no Discriminación y Accesibilidad Universal de 2003), todavía no se 
puede hablar de una accesibilidad generalizada en las páginas Web de las universidades, que continúan siendo un espacio de difícil uso para las personas con discapacidad.

Además de las páginas Web, otro aspecto de especial relevancia en el campo educativo, como se ha apuntado, son las plataformas de aprendizaje. Sobre esta cuestión no existen muchas investigaciones. Cabe destacar a Hinn (1999), que estudió cuatro entornos de accesibilidad de las personas con discapacidad a estos nuevos espacios de aprendizaje. En este estudio, contó con aportaciones de once estudiantes universitarios, que evaluaron estas plataformas. Entre sus valoraciones resaltan como problemas más relevantes la falta de textos alternativos, utilización de applets y marcos, iconos gráficos, tablas y códigos específicos de los navegadores, etc. Rowland (2000) determinó que tan sólo un $24 \%$ de las páginas Web de proveedores de educación a distancia online eran accesibles. En el contexto nacional, Gutiérrez y Restrepo y Martínez (2007), concluyen que actualmente no existe en el mercado ninguna plataforma que cumpla con los requisitos técnicos de accesiblidad, afirmación ratificada por estudios posteriores que reflejan un bajo porcentaje de éxito $(20,76 \%)$ en el cumplimiento de los criterios técnicos de los portales y plataformas de e-Learning analizadas (Observatorio de Infoaccesibilidad, 2008).

Por otro lado, estos datos reflejan que las políticas universitarias en torno a la accesibilidad distan mucho de ser las correctas. Así lo ratifican Bradbard, Peter y Caneva (2011), que concluyen que no sólo no son desarrolladas de manera deficiente, sino que en ocasiones, llegan a entrar en situaciones de ilegalidad frente a la normativa nacional existente.

Esta dualidad de las tecnologías constituye el punto de partida del presente estudio, que trata de identificar el rol que juegan las TIC en el desarrollo de la vida académica de los estudiantes con discapacidad en la universidad, con el fin de determinar en qué medida y bajo qué circunstancias constituyen una barrera o un apoyo para dicho colectivo. Esta finalidad se concreta en los siguientes objetivos específicos:

- Identificar qué uso hacen de la tecnología los estudiantes con discapacidad para establecer en qué medida las TIC constituyen una herramienta en su proceso de aprendizaje.

- Establecer las claves para diseñar una estructura tecnológica en forma de servicios de apoyo en la que los EcD encuentren las herramientas técnicas necesarias para desarrollar una vida académica y social en igualdad de condiciones en la Universidad. 


\section{Metodología y diseño de investigación}

El presente estudio forma parte de una investigación más completa financiada por la Dirección General de Universidades del Ministerio de Educación ${ }^{1}$ sobre los servicios de apoyo tecnológico y didáctico para mejorar la accesibilidad de la enseñanza universitaria para las personas con discapacidad. En dicha investigación participaron seis universidades públicas españolas: Universidad Autónoma de Madrid, Universidad de Alcalá, Universidad de Almería, Universidad de Cádiz, Universidad de Santiago de Compostela y Universidad Complutense de Madrid.

El estudio es una investigación cualitativa de tipo etnográfico exploratoria, dirigida a generar información descriptiva e interpretativa de este grupo y del sistema en el que se enmarca su actividad. De acuerdo con los objetivos propuestos, se ha realizado una recogida de información enfocada a recopilar las opiniones, valoraciones y propuestas de los estudiantes con discapacidad, utilizando un cuestionario y entrevistas semiestructuradas respectivamente como instrumentos de recogida de información. Si bien podía resultar algo rígido en su estructura, el cuestionario ha permitido posteriormente un análisis cuantitativo, así como la comparabilidad de las respuestas (Stoetzel y Girarad, 1973, citado en García Ferrando, Ibáñez y Alvira, 2005: 180).

Los datos cuantitativos recogidos de los estudiantes con discapacidad se han tratado con el programa de análisis estadístico SPSS versión 1.17.0., y los datos de tipo cualitativo con el programa HyperResearch. Se examinaron los resultados obtenidos en ambos casos y se complementó el análisis con información cuantitativa y cualitativa obtenida en la aplicación de encuestas y de análisis documental.

En el estudio realizado, se ha contado con una muestra aleatoria incidental de 87 estudiantes con discapacidad con la siguiente distribución entre las universidades participantes:

\begin{tabular}{|l|c|c|}
\hline Universidades & Frecuencia & Porcentaje \\
\hline Universidad Autónoma de Madrid. UAM & 7 & 8,0 \\
Universidad de Alcalá de Henares. UAH & 13 & 14,9 \\
Universidad de Almería. UAL & 14 & 16,1 \\
Universidad de Cádiz. UCA & 10 & 11,5 \\
Universidad de Santiago de Compostela. USC & 15 & 17,2 \\
Universidad Complutense de Madrid. UCM & 28 & 32,2 \\
Total & 87 & 100,0 \\
\hline
\end{tabular}

Tabla 1. Distribución de la muestra de EcD por universidades

\footnotetext{
${ }^{1}$ Los resultados presentados en este artículo proceden del proyecto Servicios de apoyo tecnológico y didáctico para mejorar la accesibilidad de la enseñanza universitaria para las personas con discapacidad. Dir. C. Alba Pastor. Universidad Complutense de Madrid. Este proyecto fue financiado por la Dirección General de Universidades dentro del Programa de Estudios y Análisis. Ref. EA2008-0318
} 


\section{Descripción de la muestra de estudiantes con discapacidad}

Como ya se ha indicado, se ha trabajado con una muestra aleatoria incidental, no representativa, constituida por 87 estudiantes con discapacidad que han completado correctamente el cuestionario. El porcentaje que componen la muestra por sexos evidencia una mayor participación femenina $(59,5 \%$ de mujeres frente al $40,5 \%$ de hombres).

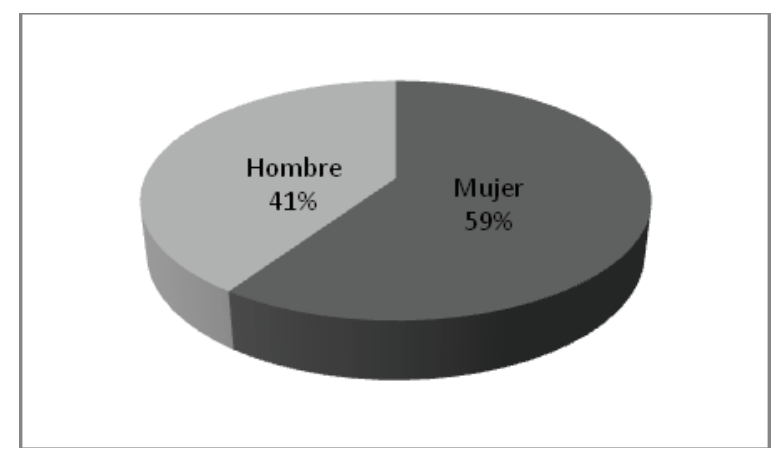

Gráfico 1. Distribución de la muestra por sexos

En cuanto al área de conocimiento, los datos muestran una clara preferencia de los estudiantes por las carreras de ramas sociales, económicas y jurídicas $(55,2 \%)$, y se evidencia un número de estudiantes mucho menor en carreras de CC. Experimentales y Técnicas $(7,1 \%$ y $4,7 \%)$

El rango de edad es muy amplio, oscilando entre los 18 y los 54 años, con una media de 28 años. La mitad de la muestra se encuentra entre los 19 y los 26 años, siendo 23 la edad del grupo participante principal. No obstante, un $44 \%$ supera los 26 años. Este dato es significativo porque manifiesta una clara presencia de estudiantes con discapacidad en las aulas universitarias a edades en las que teóricamente deberían haber finalizado sus estudios (suponiendo que cursan carreras de entre 3 y 5 años de duración, y que ingresan en la universidad aproximadamente a los 18 años). El dato resulta aún más significativo cuando observamos que un $19 \%$ de la muestra supera los 30 años de edad. Estos datos y la media de edades evidencian que ésta es superior entre los alumnos con discapacidad respecto al resto de sus compañeros universitarios.

De la muestra recogida, el 50,6\% de los estudiantes presentan una discapacidad motórica, seguido de un 18,5\% con discapacidad auditiva y un 13,6\% con discapacidad visual. El resto de casos están afectados por otro tipo de discapacidad asociada a enfermedad mental, síndrome, etc. En las estadísticas generales de la muestra, se presenta un importante grupo que corresponde al 17,3\% del total, con "otras discapacidades y necesidades". En este estudio, se ha logrado concretar y especificar algo más ese "otras": un 9,9\% presenta una discapacidad múltiple, un $3,7 \%$ tiene una enfermedad mental, un 2,5\% diferentes síndromes. 

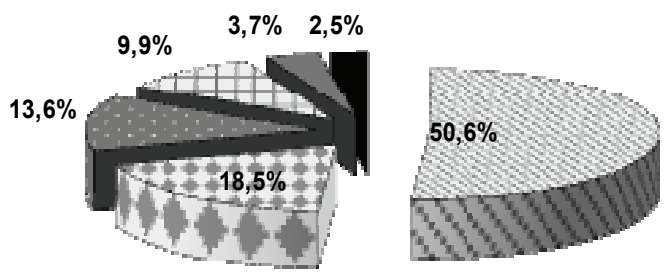

\begin{tabular}{|l|}
\hline$\square$ Motórica \\
$\square$ Auditiva \\
$\square$ Visual \\
$\square$ Múltiple \\
$\square$ Enfermedad mental \\
$\square$ Síndromes
\end{tabular}

Gráfico 2. Distribución de la muestra por tipo de discapacidad.

Observamos un grado de discapacidad bastante elevado, con una media de un $60 \%$ $(59,92 \%)$ de discapacidad reconocida. Los porcentajes oscilan entre el 22 y el $98 \%$, siendo el $65 \%$ el grado de discapacidad más frecuente (un $12 \%$ de la muestra), seguido del $33 \%$ (8\% de la muestra). La distribución según el grado de discapacidad es muy dispersa. Sin embargo, como ya se ha comentado, la media se sitúa en un $60 \%$ de grado de discapacidad.

La media de edad de inicio en el sistema educativo está por debajo de los 4 años ( $\overline{\mathbf{X}}=3,95)$, siendo los 3 años y los 4 años las edades más habituales de entrada en el sistema escolar y con los mismos porcentajes, respectivamente $(34,6 \%)$. Los datos de la muestra ponen de manifiesto que la integración de los estudiantes con necesidades de apoyo específico en el sistema educativo responde al modelo educativo normalizado. Algo más de un $75 \%$ ingresa antes de los 5 años, lo cual se corresponde con las edades habituales de entrada en el sistema educativo de cualquier niño. Por otra parte, solamente un 9,9\% lo hizo a los 6 años, mientras que un 11,1\% empezó a los 5 años. Algo más de 20 años es la media de años de permanencia en el sistema educativo de la muestra analizada $(\overline{\mathbf{X}}=20,68)$. Este dato se corresponde con el porcentaje más alto de los estudiantes entrevistados que llevan 20 años $(15,2 \%)$, seguido de los que llevan 16 años $(11,4 \%)$ en el sistema educativo. La mayoría de los estudiantes entrevistados ha cursado los estudios de Educación Primaria en un centro ordinario (86 $\%)$. Este porcentaje aumenta considerablemente al tratarse de Educación Secundaria, alcanzando el $93 \%$ de la muestra que optó cursar dichos estudios en un centro ordinario frente a otro de integración o específico.

Evidenciamos de nuevo que los estudiantes con discapacidad de la muestra han seguido una trayectoria educativa normalizada. La edad de entrada en el sistema educativo es la establecida no sólo por ley (que determina la edad obligatoria de escolarización a los seis años), sino la que socialmente está designada, y que adelanta a los tres años la entrada en el sistema educativo. Por otro lado, los estudios de Educación Primaria se han desarrollado en un porcentaje altísimo en centros 
ordinarios, siendo incluso los porcentajes más bajos registrados los correspondientes a la asistencia a centros específicos de Educación Especial (5,8 \%).La opción por un centro ordinario se acentúa aún más en la Educación Secundaria, en la que también desciende la alternativa del centro específico (tan sólo un 2,3 \%).

El rango de tiempo de permanencia dentro de la universidad oscila entre 1 y 16 años. Algo más de 5 años es la media de permanencia actual de los estudiantes con discapacidad entrevistados ( $\overline{\mathbf{X}}=5,42$ ). Un $16,9 \%$ lleva 4 y 5 años, respectivamente, en la universidad, seguido de un $13 \%$ que lleva 3 y un $7,8 \%$ con 8 años dentro del sistema universitario. Sin embargo, hay que destacar que un $33,8 \%$ de la muestra encuestada lleva entre 6 y 16 años dentro del sistema universitario, de la cual el 9,1\% está desde hace más de 10 años. Si tenemos en cuenta que la duración media de los estudios universitarios oscila entre tres y cinco años, estos datos resultan relevantes, ya que ponen de manifiesto que una parte significativa de los estudiantes con discapacidad invierten más años de los establecidos en finalizar sus estudios universitarios, en muchos casos más del doble. Las causas de este hecho debemos buscarlas en los posteriores análisis de los datos, al identificar las barreras con las que se enfrentan los estudiantes con discapacidad en el desarrollo de su vida universitaria y del proceso de enseñanza / aprendizaje.

Las conclusiones anteriores toman aún más fuerza al contrastar que un elevado porcentaje $(70 \%)$ de los alumnos con discapacidad manifiestan que su asistencia a la universidad ha sido de manera continuada.

\section{Resultados}

Los resultados que ahora se presentan se centran en los datos proporcionados sobre las tecnologías utilizadas por los estudiantes con discapacidad, quién proporciona estas tecnologías y para qué las utilizan. En otro grupo de cuestiones se analizan la utilización por estos estudiantes de la página web y del campus virtual de la universidad y las dificultades encontradas en sus respectivos manejos.

\section{- Utilización de las tecnologías}

Las tecnologías de la información y de la comunicación son un elemento básico, fundamental, común en la actividad de los jóvenes y esta presencia se ha extendido a la actividad académica. La información recogida sobre las tecnologías utilizadas por los estudiantes con discapacidad $(\mathrm{EcD})$ presentada en la Tabla 2, -en la que el valor 0 indica que no se usa y el valor 5 , un uso muy frecuente-, muestra que una gran mayoría utiliza las tecnologías de propósito general como el ordenador y el teléfono móvil y, algo menor, la impresora de tinta y el MP3. 


\begin{tabular}{|l|l|l|l|l|l|l|l|l|}
\hline \multirow{2}{*}{$\begin{array}{l}\text { Qué tecnologías } \\
\text { usan }\end{array}$} & \multicolumn{7}{|c|}{ Grado de Uso } & \multicolumn{2}{|c|}{} \\
\cline { 2 - 10 } & $\mathbf{0}$ & $\mathbf{1}$ & $\mathbf{2}$ & $\mathbf{3}$ & $\mathbf{4}$ & $\mathbf{5}$ & $\overline{\mathbf{X}}$ & $\mathbf{S}$ \\
\hline Ordenador & 1,2 & 1,2 & 4,7 & 5,8 & 7,0 & 80,2 & $\mathbf{4 , 5 7}$ & 1,01 \\
\hline Teléfono móvil & 2,4 & & 4,8 & 9,5 & 15,5 & 67,9 & $\mathbf{4 , 3 9}$ & 1,09 \\
\hline Impresora tinta & 4,9 & 3,7 & 9,8 & 8,5 & 15,9 & 57,3 & $\mathbf{3 , 9 9}$ & 1,47 \\
\hline MP3 & 30,4 & 3,8 & 5,1 & 8,9 & 8,9 & 43,0 & $\mathbf{2 , 9 1}$ & 2,19 \\
\hline Escáner & 30,8 & 6,4 & 17,9 & 14,1 & 6,4 & 24,4 & 2,32 & 1,95 \\
\hline Lector de pantalla & 80,9 & & 1,5 & 2,9 & 1,5 & 13,2 & 0,84 & 1,79 \\
\hline Navegador de Voz & 89,2 & 1,5 & 1,5 & 3,1 & 1,5 & 3,1 & 0,35 & 1,12 \\
\hline Impresora Braille & 93,4 & & & 1,6 & 1,6 & 3,3 & 0,28 & 1,08 \\
\hline Agenda Parlante & 95,4 & & 1,5 & 1,5 & & 1,5 & 0,15 & 0,75 \\
\hline
\end{tabular}

Tabla 2. Qué tecnologías utilizan los EcD

La tecnología más utilizada, con una media de 4,57 sobre 5, es el ordenador, obteniendo la mayor frecuencia $(80,2 \%)$ en el máximo valor. Es decir, que la mayoría de los estudiantes dicen utilizar el ordenador y lo hacen con mucha frecuencia. Los valores obtenidos en todas las demás categorías de menor frecuencia son muy inferiores. Algo similar ocurre en el caso del teléfono móvil, que también lo utiliza una mayoría de casi el 70\% de los encuestados, con un patrón similar. Es decir, lo utilizan muchos y lo utilizan con mucha frecuencia. A cierta distancia, pero con el mismo patrón, con una frecuencia del 57,3\% aparece la utilización de la impresora de tinta. Distinto patrón se encuentra en la utilización del MP3, que aunque un 43\% lo utiliza mucho, hay un $30 \%$ que no lo usa nada.

Mención aparte merecen la utilización de las tecnologías específicas, como son la Impresora Braille, Lector de pantalla, Navegador de voz y la Agenda parlante, especialmente utilizadas por los estudiantes con discapacidad visual. Dentro de este grupo, el recurso más utilizado es el lector de pantalla, utilizado con mucha frecuencia por un $13 \%$ de este grupo de encuestados. 


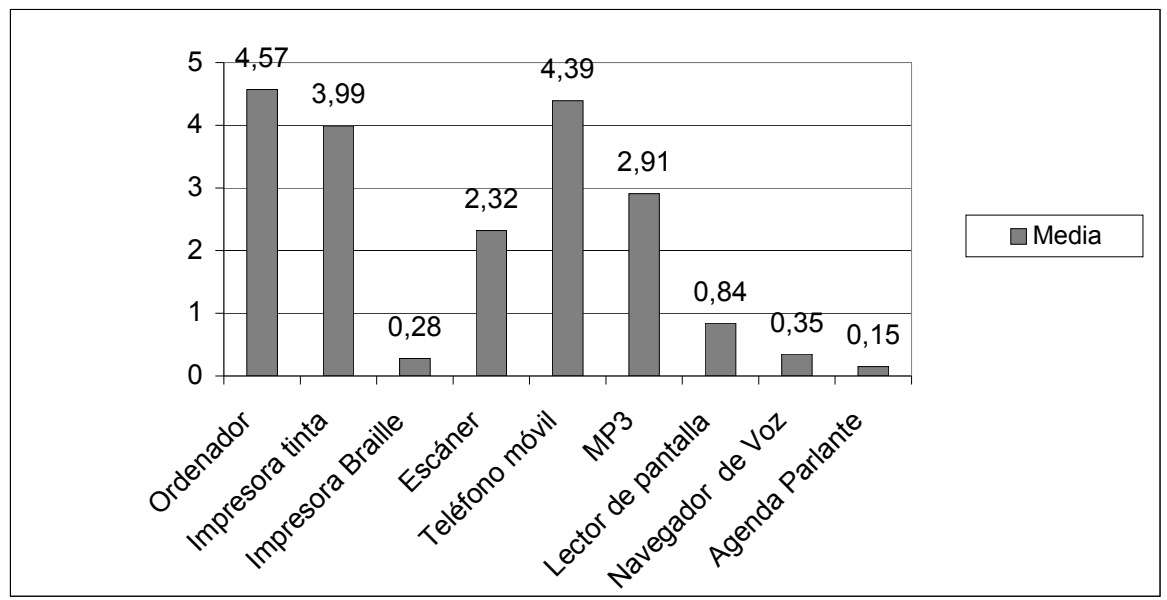

Gráfico 3. Qué tecnologías utilizan los EcD

En el Gráfico 3 se observa con claridad que entre los EcD existe un alto nivel de uso de recursos tecnológicos, con un patrón de utilización generalizada de herramientas como el ordenador, el teléfono móvil, la impresora y el MP3, que utilizan la mayoría y lo hacen con mucha frecuencia. Un patrón que es común entre todos los estudiantes universitarios, como mostraba en sus estudios Fichten (2005 y 2010), en los que las tecnologías son facilitadores de la actividad académica, para los que tienen y los que no tienen discapacidades.

\section{- Quién proporciona estas tecnologías}

Un aspecto que resulta de interés es la provisión de dichas tecnologías. Los datos recogidos entre los EcD indican que estas tecnologías, que tienen un papel fundamental para permitirles participar en la actividad académica, en la mayoría de los casos $(70,30 \%)$ son los propios estudiantes los que tienen que adquirir o conseguir estos recursos.

\begin{tabular}{|l|c|c|}
\hline & Frecuencia & Porcentaje \\
\hline Universidad & 11 & 10,89 \\
Otra entidad & 19 & 18,81 \\
Uno mismo & $\mathbf{7 1}$ & $\mathbf{7 0 , 3 0}$ \\
\hline
\end{tabular}

Tabla 3. Provisión de las tecnologías.

Una pequeña parte, casi un $19 \%$ de las respuestas indican que son otras entidades las que proporcionan estas tecnologías. Es el caso de la ONCE, que proporciona tecnologías específicas al propio estudiante o a través de puestos adaptados en los centros (aulas y bibliotecas). Pese a que la Ley 51/2003, de 2 de diciembre, de igualdad de oportunidades, no discriminación y accesibilidad universal de las personas con discapacidad, dispone que sean las instituciones las que deben proveer de "apoyos 
complementarios, tales como ayudas económicas, tecnológicas de apoyo, servicios o tratamientos especializados y otros servicios personales. En particular, ayudas y servicios auxiliares para la comunicación, como sistemas aumentativos y alternativos, sistemas de apoyos a la comunicación oral y lengua de signos u otros dispositivos que permitan la comunicación" (Artículo 10.2), los datos según la muestra encuestada es que sigue siendo, de forma mayoritaria, el propio EcD quien asume la adquisición y provisión de estas tecnologías y recursos.

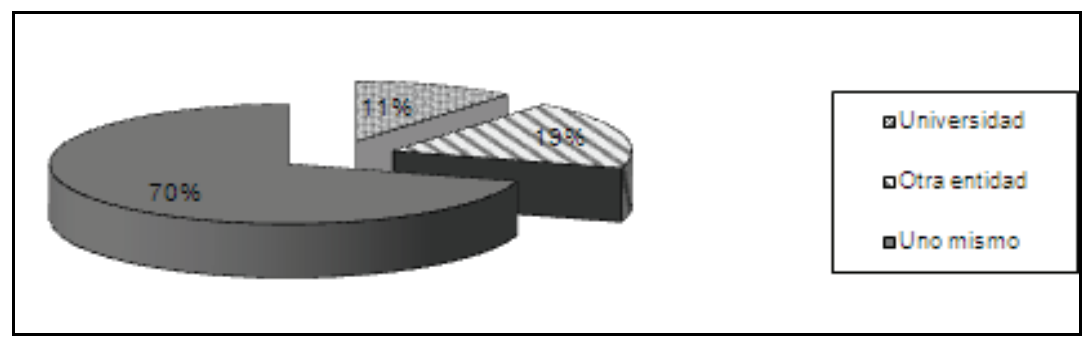

Gráfico 4. Las tecnologías facilitadas a los EcD según su proveedor

Estos resultados hacen aflorar una cuestión controvertida sobre si deben ser las instituciones quienes deban asumir la diversidad de sus estudiantes y garantizar estos recursos a los EcD o es la discapacidad un problema del estudiante, no del sistema universitario, y por ello deben ser los estudiantes quienes se ocupen de conseguirlos.

\section{- Con qué finalidad se utilizan las TIC}

Si las tecnologías de la información y la comunicación son utilizadas por los universitarios y constatado que dentro de este universo, los EcD también hacen un uso frecuente de las mismas, se trataba de indagar sobre el tipo de uso que estos estudiantes hacen de ellas, y si prevalece algún uso, más de tipo comunicativo, instrumental o de carácter académico investigador.

\begin{tabular}{|l|c|c|}
\hline & Frecuencia & Porcentaje \\
\hline Apoyo en clase & 33 & 40,2 \\
Estudiar y hacer trabajos & $\mathbf{7 9}$ & $\mathbf{9 6 , 3}$ \\
Búsqueda de materiales & $\mathbf{7 7}$ & $\mathbf{9 3 , 9}$ \\
Comunicación con lo compañeros & $\mathbf{7 1}$ & $\mathbf{8 6 , 6}$ \\
Comunicación con los profesores & $\mathbf{7 0}$ & $\mathbf{8 5 , 4}$ \\
\hline
\end{tabular}

Tabla 4. Finalidad con la que se utilizan las TICs por los Ecd

Los datos obtenidos mostrados en la Tabla 4, sobre la finalidad de la utilización de las tecnologías reflejan dos tipos de usos mayoritarios, con valores muy elevados, por encima del $85 \%$ en todos los casos. En primer lugar se utilizan para tareas académicas, 
como el estudio y realización de trabajos $(96,3 \%)$ y la búsqueda de materiales $(93,9 \%)$. El segundo grupo hace referencia a la utilización de estos recursos para facilitar la comunicación de los EcD con sus compañeros y profesores. Estos valores contrastan con la puntuación mucho menor que recibe la utilización como apoyo en las actividades del aula.

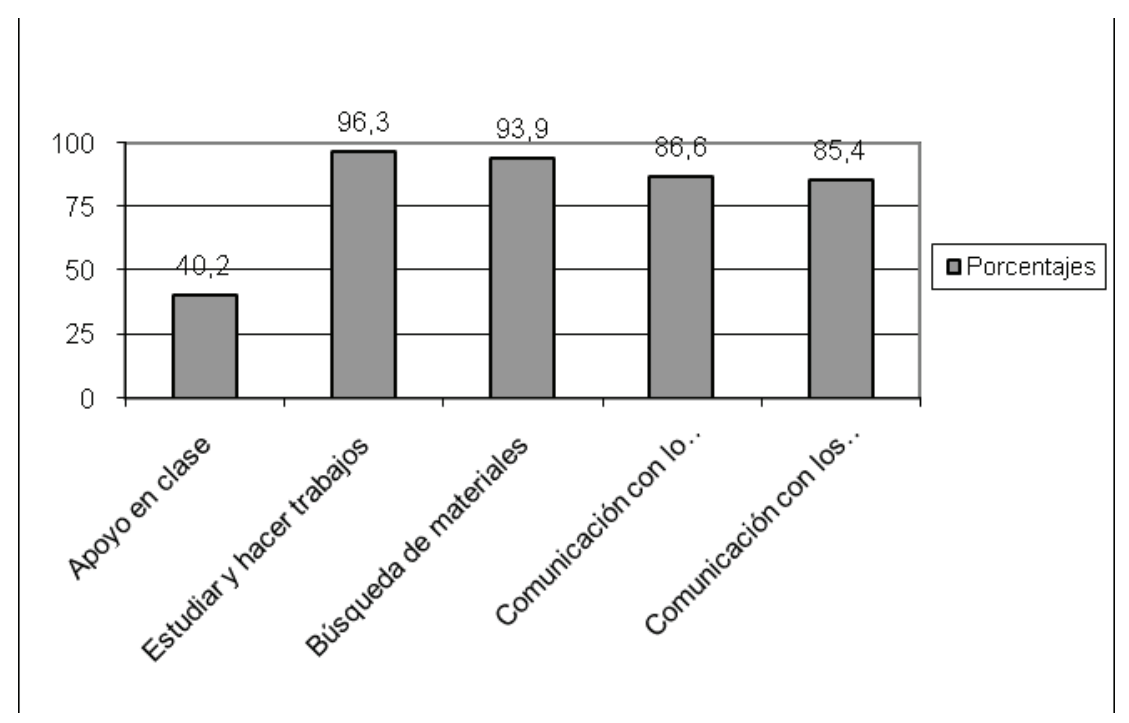

Gráfico 5. Tipo de uso de las TICs que realizan los EcD

Como se aprecia en el Gráfico 5, hay una utilización casi uniforme en cuanto a al aprovechamiento de las tecnologías para las actividades relacionadas con el estudio y realización de trabajos, junto a la búsqueda de información y materiales. Las TICs se han convertido en una herramienta indispensable en la actividad académica. Pero también hay una utilización vinculada a los procesos de comunicación entre estudiantes y de los estudiantes con sus profesores, para tareas de tutoría. Frente a esta utilización más de carácter instrumental se aprecia un notable descenso en lo que se refiere a la presencia de estos recursos como apoyo en el aula en los procesos de enseñanza- aprendizaje.

\section{- Página web de la Universidad}

Todas las universidades han invertido notables esfuerzos para tener una tarjeta de presentación en sus respectivas páginas web, con un importante componente informativo, pero también interactivo para la comunidad universitaria, en la realización de gestiones, información, comunicación y actividades formativas a través de los campus virtuales, a los que se accede a través de estas páginas. 
Al preguntar a los estudiantes de la muestra sobre los usos que hacían de la página web de su universidad las respuestas, como se refleja en la Tabla 5, muestran que el mayor porcentaje se refiere a su utilización para acceder al Campus virtual $(38,50 \%)$ seguido de las consultas de tipo informativo $(36,30 \%)$, porcentajes muy por encima de otro tipo de consultas de tipo administrativo, como es el caso de la consulta del expediente académico (16,30\%), o muy por debajo, las consultas a la biblioteca, que solo realiza un $8,90 \%$ de los encuestados.

\begin{tabular}{|l|c|c|}
\hline & Frecuencia & Porcentaje \\
\hline Campus virtual & $\mathbf{5 2}$ & $\mathbf{3 8 , 5 0}$ \\
Información general de la universidad & $\mathbf{4 9}$ & $\mathbf{3 6 , 3 0}$ \\
Consulta de expediente & 22 & 16,30 \\
Biblioteca & 12 & 8,90 \\
\hline
\end{tabular}

Tabla 5. Finalidad con la que se utiliza la página web de la universidad

Estos resultados remarcan la importancia que ha adquirido en la enseñanza universitaria la actividad a través de las plataformas virtuales, los llamados campus virtuales, cada vez más presentes en la actividad académica, incluso, como es el caso, de las universidades que imparten su enseñanza siguiendo el modelo presencial. Las funcionalidades de estas tecnologías, facilitando la información, los repositorios de documentos, la realización de actividades on-line y la comunicación entre estudiantes y profesores, han hecho de ellos un recurso cada vez más necesario y útil en los sistemas de enseñanza presencial, que van configurándose como fórmulas de enseñanza mixta $o$ Blended-learning, en las que la enseñanza presencial encuentra un gran aliado y apoyo en los servicios de las plataformas virtuales.

La riqueza de información que aparece en las páginas web de las instituciones las han convertido en una herramienta de gran utilidad, un lugar obligado de consulta, desde convocatorias, horarios, exámenes, localización, planes de estudios, actividades académicas,... Las páginas web de las universidades se han convertido en una réplica informativa de las mismas, a las que se tiene en cualquier momento fácil acceso, lo que las hace más conveniente para los estudiantes que pueden tener dificultades en la movilidad o para realizar consultas telefónicas. En esta misma línea, las respuestas de los estudiantes constatan el valor de la página web de la universidad para facilitar las gestiones administrativas $\mathrm{y}$, más concretamente, todo lo relativo a la consulta del expediente. 


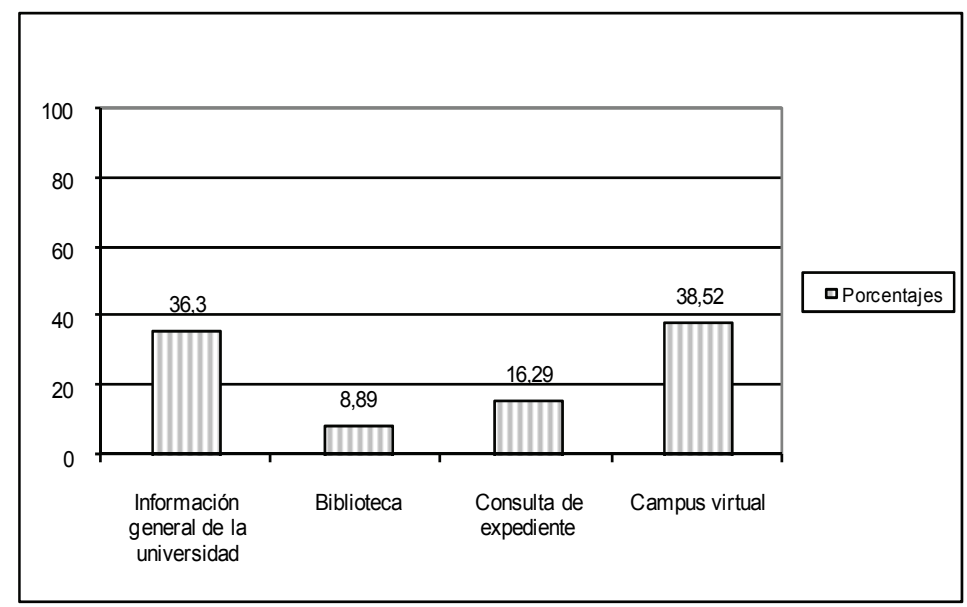

Gráfico 6. Utilización de la página web de la universidad

Por otra parte, tal y como se muestra en el Gráfico 6, sorprende el bajo resultado que obtiene el uso de la Biblioteca a través de Internet. Cabría esperar que para los EcD este fuera un servicio de gran utilidad, por facilitar el acceso a numerosos recursos bibliográficos, el acceso a bases de datos y documentos on-line que ofrecen la mayoría de ellas. Esta baja utilización puede deberse a que muchos de los documentos que se utilizan para las clases se encuentran en el campus virtual, cuando se trata de documentos en formato digital, o cuando no son digitales tienen que acudir personalmente a la biblioteca.

Consultados sobre posibles dificultades encontradas para acceder a la página web de su universidad, la mayoría los estudiantes con discapacidad (EcD) no encuentra ningún problema (53.4\%). En los datos de la Tabla 6 se aprecia que es accesible para 3 de cada 4 estudiantes. No obstante, sí hay estudiantes que indican tener bastantes o muchos problemas $(23,3 \%)$, y que requeriría atención por parte de los responsables de las páginas web de las universidades, para evitar que estos problemas se añadan a otros previos.

\begin{tabular}{|l|l|c|c|}
\hline \multicolumn{2}{|c|}{} & Frecuencia & Porcentaje \\
\hline & 0- Ningún problema & $\mathbf{3 9}$ & $\mathbf{5 3 , 4}$ \\
& 1- Muy pocos problemas & 4 & 5,5 \\
$\mathbf{X}=\mathbf{1 , 6 0}$ & 2- Pocos problemas & 4 & 5,5 \\
$\mathbf{S}=\mathbf{1 , 9 5}$ & 3- Algunos problemas & 9 & 12,3 \\
& 4- Bastantes problemas & 7 & 9,6 \\
& 5- Muchos problemas & 10 & 13,7 \\
\hline
\end{tabular}

Tabla 6. Dificultades en el uso de la página web de la universidad. 
Estas dificultades son especialmente importantes cuando se compromete la posibilidad de realizar las actividades académicas a través del campus virtual. Ante estas dificultades, los propios estudiantes buscan soluciones a través de otros recursos, personales y tecnológicos.

"No pone todas las informaciones necesarias en la página y lo soluciono comunicando con mis compañeros sea por Internet o por sms" (UCA01)

"La verdad es que [termino] agobiándome, porque hay veces que no encuentro las cosas y al final desisto y no continuo" (UAL05).

"Los resuelvo yo, dedicándole más tiempo"(UAM02).

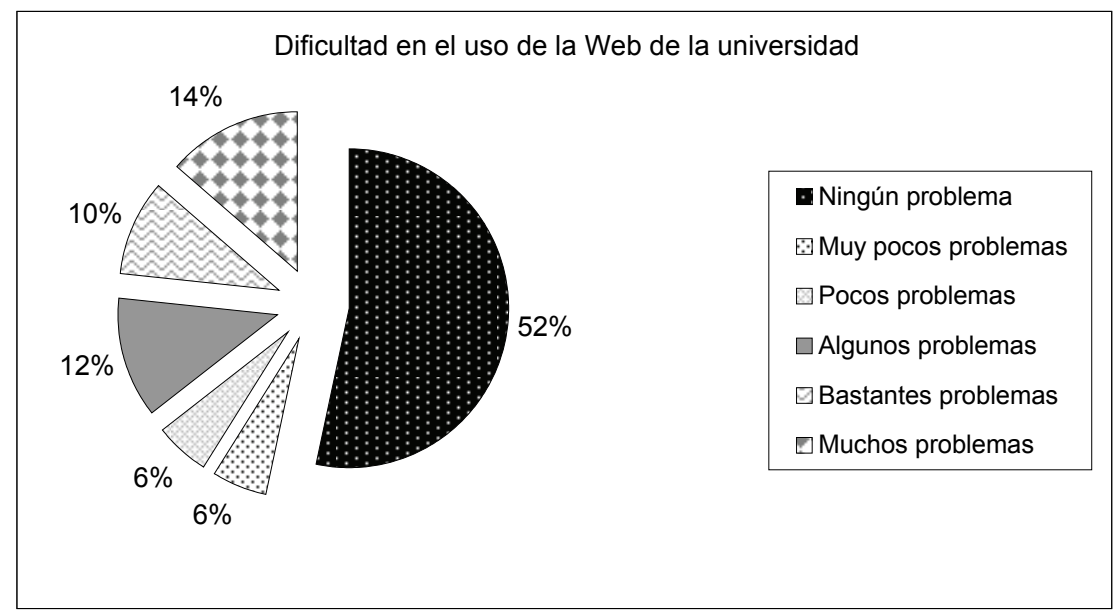

Gráfico 7. Dificultades encontradas en la página web de la universidad por parte de los EcD

\section{- Utilización del Campus Virtual}

Más de la mitad de los estudiantes con discapacidad encuestados cursan materias apoyadas en plataformas virtuales de enseñanza (59,7\%), los llamados "campus virtuales" de cada universidad. El número de asignaturas varía. Entre los estudiantes de la muestra el número de asignaturas cursadas a través o con apoyo del campus virtual de su universidad oscila entre 0 y 8 , como queda recogido en la siguiente tabla. 


\begin{tabular}{|l|c|c|c|}
\hline \multicolumn{2}{|c|}{ Campus Virtual } & Frecuencias & Porcentaje \\
\hline & 0 & 4 & 8,2 \\
Datos sobre los casos que & 1 & 5 & 10,2 \\
sí utilizan el Campus & $\mathbf{2}$ & $\mathbf{8}$ & $\mathbf{1 6 , 3}$ \\
Virtual n=52 & $\mathbf{3}$ & $\mathbf{7}$ & $\mathbf{1 4 , 3}$ \\
& 4 & 6 & 12,2 \\
& $\mathbf{5}$ & $\mathbf{8}$ & $\mathbf{1 6 , 3}$ \\
& 6 & 4 & 8,2 \\
& 7 & 1 & 2 \\
& 8 & 6 & 12,2 \\
\hline
\end{tabular}

Tabla 7. Número de materias cursadas por los EcD en las que utilizan el campus virtual

La importancia de estos resultados radica en su relación con los datos de accesibilidad que aparecían en la pregunta anterior. Es decir, hay una tendencia a utilizar los campus virtuales para impartir asignaturas o como apoyo a asignaturas que se imparten de manera presencial. Si estas páginas no son accesibles para los EcD, estos no podrán participar en actividades que se realicen en estos contextos virtuales, ni realizar las actividades de aprendizaje o tener acceso a las informaciones, la comunicación o los materiales que estén disponibles en los espacios virtuales para los estudiantes de un grupo. De ello se deriva la trascendencia de garantizar la accesibilidad de estos entornos y su contenido para evitar dificultades en el proceso de enseñanza y, con ello, de exclusión educativa.

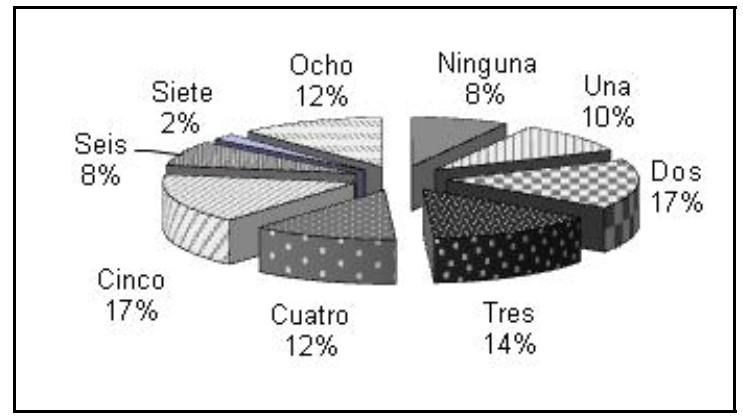

Gráfico 8. Materias cursadas con uso del campus virtual

Consultados los EcD de la muestra sobre las dificultades encontradas en la utilización del campus virtural de su universidad, los datos muestran unos índices muy bajos en la percepción de dificultades en la mayoría de los servicios que se utilizan dentro de estas plataformas. En todos los casos, en una escala entre los valores 0 y 5 , 
donde 0 es "ningún problema" y 5 "muchos problemas", más del $80 \%$ de las respuestas se encuentran en el valor 0 .

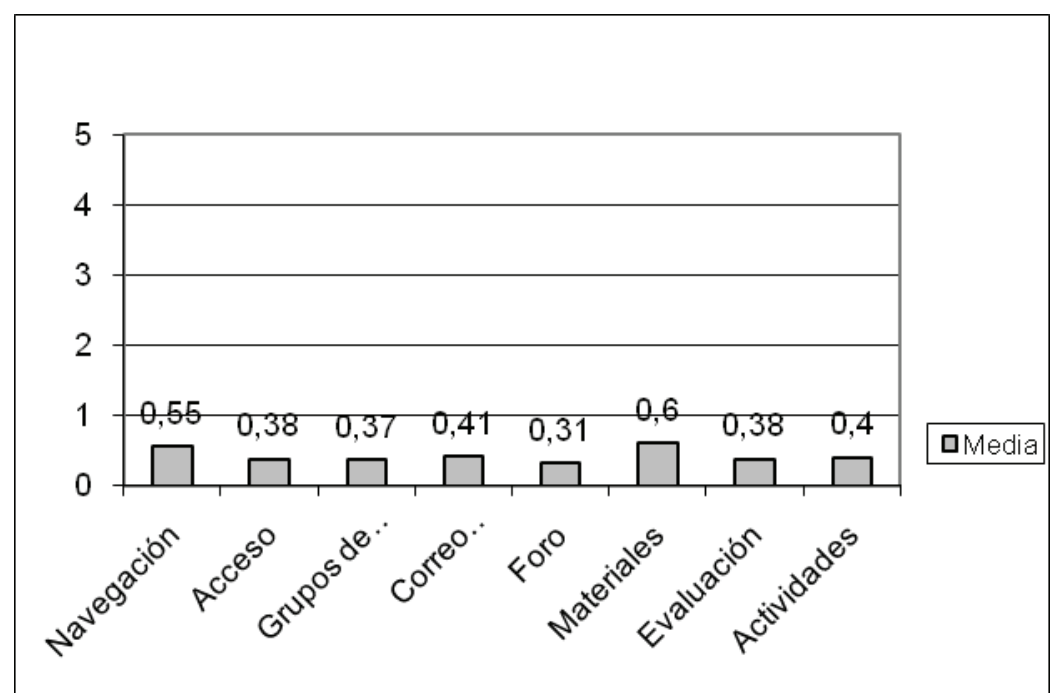

Gráfico 9. Valoración media de los problemas detectados en el uso del Campus Virtual

Analizando los datos recogidos mostrados en la Tabla 8, -en la que el valor 0 indica que no se encuentran problemas y el valor 5 , muchos problemas- , cuando se agrupan los valores en las puntuaciones más altas ( 4 y 5 ) se observa que un $10 \%$ de los EcD encuentran muchos problemas con los materiales que los docentes utilizan o ponen a disposición de los estudiantes de una materia en el campus virtual. En el resto de las categorías, en la mayoría de los servicios hay un porcentaje en torno al $6 \%$ que también encuentra muchas dificultades, como son la navegación, acceso o la evaluación en estos entornos virtuales.

\begin{tabular}{|l|l|l|l|l|l|l|l|l|}
\hline & \multicolumn{7}{|c|}{ Grado } & \multicolumn{1}{l|}{} \\
\cline { 2 - 10 } & $\mathbf{0}$ & $\mathbf{1}$ & $\mathbf{2}$ & $\mathbf{3}$ & $\mathbf{4}$ & $\mathbf{5}$ & $\mathbf{X}$ & $\mathbf{S}$ \\
\hline Navegación & $\mathbf{8 1 , 6}$ & 2,0 & 4,1 & 6,1 & 4,1 & 2,0 & 0,55 & 1,27 \\
\hline Correo electrónico & $\mathbf{8 3 , 7}$ & 2,0 & 8,2 & 2,0 & 4,1 & & 0,41 & 1,01 \\
\hline Actividades & $\mathbf{8 6 , 0}$ & 2,3 & 2,3 & 4,7 & 4,7 & & 0,40 & 1,07 \\
\hline Acceso & $\mathbf{8 4 , 9}$ & 3,8 & 5,7 & & 5,7 & & 0,38 & 1,02 \\
\hline Evaluación & $\mathbf{8 6 , 7}$ & 4,4 & 2,2 & & 4,4 & 2,2 & 0,38 & 1,13 \\
\hline Grupos de Trabajo & $\mathbf{8 9 , 1}$ & & 2,2 & 4,3 & 2,2 & 2,2 & 0,37 & 1,12 \\
\hline Foro & $\mathbf{9 1 , 1}$ & & 2,2 & 2,2 & 2,2 & 2,2 & 0,31 & 1,06 \\
\hline Materiales & $\mathbf{8 2 , 0}$ & 4,0 & 2,0 & 2,0 & $\mathbf{4 , 0}$ & $\mathbf{6 , 0}$ & 0,60 & 1,45 \\
\hline
\end{tabular}

Tabla 8. Problemas con el uso del Campus Virtual 
En relación a estos problemas, las respuestas recogidas indican que en la mayoría de casos tienen que ver con la estabilidad de la aplicación (se bloquea), la incompatibilidad de las herramientas de que disponen los entornos virtuales con los programas utilizados por los EcD y, con la poca usabilidad, es decir, facilidad de uso y amigabilidad del interfaz.

Si bien se trata de porcentajes poco elevados, es necesario seguir avanzando en la mejora de la accesibilidad de estos recursos, ya que se han convertido en un elemento fundamental en los procesos de enseñanza y para el aprendizaje de los estudiantes. Solo así se logrará garantizar que todos los estudiantes puedan utilizar y beneficiarse de estos recursos y no generar diferencias entre los que pueden acceder a ellos y los que no.

\section{Discusión}

La imagen que se desprende de los resultados presentados retrata a unos EcD universitarios que manejan las TIC con profusión y soltura en su vida cotidiana para comunicarse, informarse y para realizar actividades en el marco de las plataformas o campus virtuales. Utilizan tecnologías específicas pero, sobretodo, usan las más extendidas entre toda la población como son el ordenador, el teléfono móvil, la impresora y el MP3. Estos resultados coinciden con lo apuntado por Fichten, Asuncion, Nguyen, Budd y Amsel (2010) que encuentran en su trabajo una utilización generalizada y bastante satisfactoria de tecnologías por parte de los estudiantes universitarios con discapacidad que valoran como adecuadas a sus necesidades. Los resultados de este trabajo también coinciden con los autores citados, en que para las actividades académicas, en la mayoría de los casos, son los propios estudiantes los que se consiguen las tecnologías que les permiten participar y seguir las clases o las actividades que acompañan los procesos de enseñanza y aprendizaje. Principalmente las utilizan para comunicarse y hacer trabajos, y les sirven poco como apoyo en las clases.

Para estos estudiantes la página web de su universidad no tiene dificultades relevantes, y la utilizan para acceder al campus virtual y para informarse. Se utiliza también para trámites administrativos y, escasamente, para acceder a la biblioteca. Estos resultados difieren de los resultados obtenidos por diferentes autores en trabajos ya citados, realizados hace unos años por Flowers, Bray y Algozzine (2001), Toledo (2001), Termens y Ribera (2002), Alba, Zubillaga y Ruíz (2003) y por el Observatorio de Infoaccesibilidad $(2010,2006)$, donde el diagnóstico de las páginas web de las universidades son en su mayoría no accesibles. Parece que se ha mejorado el diseño accesible y la compatibilidad con los programas específicos que utilizan algunos estudiantes con discapacidad y cada vez son menos los problemas identificados.

Atención especial requiere la utilización del campus virtual, ya que como se ha constatado, aunque todos los estudiantes encuestados pertenecen a universidades que imparten sus enseñanzas en formato presencial, la mayoría tiene asignaturas vinculadas 
al campus virtual, con servicios como el correo electrónico, foros, grupos de trabajo, actividades, evaluación o repositorio de materiales. Los resultados obtenidos en este trabajo difieren con los resultados expuestos por Martínez (2007) ó del Observatorio de Infoaccesibilidad (2008), según los cuales las plataformas existentes en el mercado en el momento de la realización de sus estudios, no cumplían con los requisitos técnicos de accesibilidad ni con los criterios técnicos de los portales y plataformas virtuales de enseñanza. Quizás sea porque en estos años ha mejorado la accesibilidad de estas plataformas, pero los resultados obtenidos son discrepantes ya que, en la mayoría de los casos, los estudiantes del estudio señalan que apenas encuentran problemas para hacer uso en su actividad académica en estas plataformas, lo que supone una garantía para que los EcD puedan cursar sus asignaturas y participar en condiciones similares a las de sus grupos de compañeros.

En la misma línea a lo ya apuntado en otros estudios previos, como los ya citados de Gillette, (2006), Lewis, Yoder, Riley, So y Yusufali (2007); Zubillaga (2006) y Alba (2005), las universidades han avanzado en la mejora de la accesibilidad de los entornos virtuales de aprendizaje, pero todavía no se ha alcanzado la accesibilidad plena a los mismos. Existen algunos problemas, que aunque afectan de forma minoritaria, deben resolverse para garantizar la plena accesibilidad a los contextos educativos de todos los estudiantes, que están centradas en cuestiones referidas a la usabilidad del interfaz y la compatibilidad con programas específicos y ayudas técnicas utilizadas por los ECD.

\section{Conclusiones}

La utilización de las TICs se ha revelado como un componente clave de una enseñanza accesible, no sólo de aquellas que pueden y deben utilizar los docentes en sus clases teóricas y prácticas, sino también los medios y ayudas técnicas y tecnologías que utilizan los EcD para la realización de sus trabajos, la búsqueda de información o el seguimiento y la participación en las clases.

La utilización de apoyos técnicos y tecnológicos ha demostrado ser, cuando se utilizan adecuadamente, una de las mejores opciones para lograr la accesibilidad a los distintos procesos de la vida en la universidad: en los usos que pueden hacer de ellas los EcD, al ser utilizadas por los docentes en las clases, en el diseño de materiales o en la digitalización de los contenidos y aprovechamiento de espacios virtuales.; o como es el caso de la página web de las universidades, en las gestiones administrativas para acceder a la información, trámites, etc. De ahí su trascendencia de lograr que estos recursos sean elementos habituales en la actividad docente y de aprendizaje en la universidad y en garantizar su uso adecuado y la accesibilidad de su diseño.

Además de ello, se constata el progresivo acompañamiento de las actividades presenciales por recursos de respaldo en espacios del tipo Campus Virtuales, que ha dado lugar a que el profesorado proporcione una pluralidad de materiales y de recursos en la enseñanza universitaria, que se ha visto incrementado con las ventajas y la potencialidad de las TIC. Esta evolución hacia modelos mixtos de enseñanza debería acompañarse con recursos multimediales, y mejoras en la interacción entre y con los 
estudiantes, garantizando que tanto los materiales como los procesos de interacción son accesibles para todos los estudiantes, con y sin discapacidad.

En la utilización de estos espacios como repositorios de documentos y materiales no siempre se tienen en cuenta los requisitos de accesibilidad, por lo que se deriva la necesidad de promover información y formación dirigida al profesorado universitario en la creación o generación de materiales que sean accesibles, para lo que ya se cuenta con materiales específicos diseñados con esta finalidad (Zubillaga, 2008; Sánchez Hípola, 2008), así como sobre la gestión de contenidos accesibles y la utilización didáctica de los espacios virtuales, con el fin de garantizar la accesibilidad a los mismos a todos los estudiantes.

Los estudiantes encuestados utilizan estas tecnologías para hacer sus trabajos, buscar información y comunicarse. Es evidente, pues, la trascendencia que tiene para los EcD poder acceder a los materiales y contenidos de aprendizaje con la garantía de hacerlo de forma similar a como lo hacen el resto de estudiantes, en tiempo y forma, de donde se deriva la necesidad de garantizar la accesibilidad de los soportes tecnológicos y de los entornos digitales. De no ser así, tal y como se desprende del estudio, ésta sería una de las barreras identificadas por los estudiantes para poder acceder a los aprendizajes que actuaría, de facto, como una forma de discriminación indirecta.

En síntesis, los resultados del estudio sobre la utilización de las tecnologías por los estudiantes con discapacidad en las universidades, conscientes de las limitaciones derivadas de la peculiaridad de la muestra, permiten identificar algunos elementos clave en tornoo a los cuales proponer acciones que redunden en la mejora de la accesibilidad de la actividad universitaria en relación a la utilización de las TICs: el componente didáctico, la accesibilidad y la formación.

- Componente didáctico. Dado el potencial que tienen las tecnologías en los procesos de comunicación, su flexibilidad y versatilidad para presentar información utilizando diferentes formatos, las universidades deberían reforzar su función no solo como un elemento instrumental o para el almacenamiento de recursos y materiales, sino como un componente relevante en el proceso de aprendizaje: utilización de los foros para facilitar la participación de todos los estudiantes, el correo electrónico como complemento en la actividad tutorial, o actividades didácticas en las asignaturas virtualizadas.

- Accesibilidad. No basta con la existencia de las tecnologías, de los espacios de aprendizaje o de los contenidos en formato digital. Hay que garantizar que son accesibles para todos los estudiantes, con o sin discapacidad, en unos casos utilizando un diseño universal y, en otras, diferentes formatos. La accesibilidad de las tecnologías no es una cuestión simple que se pueda lograr a través de una acción concreta. Hay que garantizar la accesibilidad de las TIC en sus distintas facetas o componentes: acceso a los procesos de enseñanza aprendizaje con las tecnologías generales, específicas o ayudas técnicas; utilización de tecnologías accesibles (software, hardware, páginas web,...); materiales de aprendizaje y contenidos en formato digital accesibles $\mathrm{y}$, accesibilidad de los procesos de 
aprendizaje cuando se utilicen diferentes tecnologías (presentaciones audiovisuales subtituladas, actividades en las plataformas digitales, laboratorios, etc. ).

- Formación. Las claves anteriores requieren la promoción de una política formativa en relación con la discapacidad y las TIC entre el profesorado universitario y los propios estudiantes, que incluya el conocimiento de las ventajas y posibilidades de estas tecnologías, de los recursos disponibles y de su utilización en los procesos de aprendizaje para los estudiantes, en los de enseñanza para los docentes y administrativos para el personal de los diferentes servicios.

Las TICs son un recurso que está en la vida de los universitarios con y sin discapacidad, que son a la vez, como se ha puesto de manifiesto, fuente de unas barreras y recurso para la eliminación de otras. De ello se deriva la necesidad de conocer cómo utilizarlas para seguir avanzando en el logro de una universidad accesible.

\section{Referencias bibliográficas}

ALBA, C. (2005). Educación superior sin barreras: La accesibilidad de las universidades para los estudiantes con discapacidad. Encounters on Education, 6, Fall 2005, 43-60.

ALBA, C.; ZUBILLAGA, A. \& RUIZ N. (2003). Educación Superior y discapacidad: Accesibilidad de las páginas web de las universidades estatales. Comunicación y Pedagogía, 188, 25-30.

ARI, I.A. \& INAN, F.A. (2010). Assistive Technology for Students with Disabilities: A Survey of Access and Use on Turkish Universities. Turkish Online Journal of Educational Technology, 9 (2), 40-45.

BARFIELD, J.P.; BENNETT, J.; FOLIO, M.R. \& KILLMAN, C. (2007). Disability Rights in Higher Education: Ensuring Kinesiology Program and Accreditation Standars Do Not Discriminate. Quest, 59 (4), 384-397.

BRADBARD, D.A.; PETERS, C. \& CANEVA, Y. (2011). Web Accessibility Policies at Land-Grant Universities. Internet and Higher Education, 13 (4), 258-266.

BOURKE, A.; STREHORN, K. \& SILVER, P. (2000). Faculty Members'Provision of Instructional Accommodations to Students with LD. Journal of Learning Disabilities, 33, Jan-Feb 2000.

BURGSTAHLER, S. (2011). Universal Design: Implications for Computing Education. ACM Transactions on Computing Education, 11 (3).

FICHTEN, C.S.; JORGENSEN, S.; BARILE, M.; HAVEL, A. (2005). College Students with Disabilities. Documento ERIC ED490017. http://www.eric.ed.gov/ERICWebPortal/search/recordDetails.jsp?ERICExtS earch_SearchValue_0=EJ906700\&searchtype=keyword\&ERICExtSearch_S 
earchType_0 $=$ no\&_pageLabel $=$ RecordDetails\&accno $=$ EJ906700\&_nfls $=$ fal se\&source $=$ ae

FICHTEN, C.S.; ASUNCION, J. V.; NGUYEN, M. N.; BUDD, J.; AMSEL, R. (2010) The POSITIVES Scale: Development and Validation of a Measure of How Well the Information and Communication Technology Needs of Students with Disabilities are Being http://www.eric.ed.gov/ERICWebPortal/search/recordDetails.jsp?ERICExtSear ch_SearchValue_0=EJ906700\&searchtype $=$ keyword\&ERICExtSearch_Search Type_0=no\&_pageLabel $=$ RecordDetails\&accno=EJ906700\&_nfls $=$ false\&sour $\mathrm{ce}=\mathrm{a}-\mathrm{J}$ ournal of Postsecondary Education and Disability, v23, n.2, p.137-154

FLOWERS, C.; BRAY, M. \& ALGOZZINE, R. (2000). Content Accessibility of Community college Web Sites. Community College Journal of Research and Practice, 25 (7), 475-485.

GARCÍA FERRANDO, M; IBÁÑEZ, J. \& ALVIRA, F. (2005). El análisis de la realidad social. Madrid: Alianza

GILLETTE, Y. (2006). Assistive Technology and Literacy Partnerships. Topics in Language Disorders, 26 (1), 70-84.

GUTIÉRREZ Y RESTREPO, E. \& MARTÍNEZ, L. (2007). La accesibilidad Web. Cómo construir una Web educativa accesible. En Segura, M. (Dir.), Accesibilidad, educación y TIC. Madrid: Centro Nacional de Información y Documentación Educativa. http://ares.cnice.mec.es/informes/17/index.htm.

HINN, D. M. (1999). Evaluating the Accessibility of Web-Based Instruction for Students with Disabilities. Extraído el 4 de abril de 2008 desde http://www.eric.ed.gov/PDFS/ED436151.pdf

KONUR, O. (2006). Teaching Disabled Students in Higher Education. Teaching in Higher Education, 11 (3), 351-363.

INSTITUTO NACIONAL DE ESTADÍSTICA (2008). Encuesta sobre Discapacidad, Autonomía Personal y Situaciones de Dependencia. Madrid: Autor.

LEWIS, K.; YODER, D.; RILEY, E.; SO, Y. \& YUSAFALI, S. (2007). Accessibility of Instructional Web Sites in Higher Education. EDUCAUSE Quarterly, 30 83), 29-35.

Ley 51/2003, de 2 de diciembre, de igualdad de oportunidades, no discriminación y accesibilidad universal de las personas con discapacidad. BOE, 3 de diciembre de 2003. http://www.boe.es/boe/dias/2003/12/03/pdfs/A43187-43195.pdf

OBSERVATORIO DE INFOACCESIBILIDAD (2006). Accessibilidad de los portales Web Universitarios 2006. Extraído el 4 de abril de 2008 desde http://www.discapnet.es

OBSERVATORIO DE INFOACCESIBILIDAD (2008). Accessibilidad de los portales Web de servicios y plataformas de e-Learning. Extraído el 4 de abril de 2008 desde http://www.discapnet.es 
OBSERVATORIO DE INFOACCESIBILIDAD (2010). Accessibilidad de los portales Web Universitarios 2010. Extraído el 4 de marzo de 2011 desde http://www.discapnet.es

RAO, M.S. (2002). Students with disabilities in higher education: Faculty attitudes and willingness to provede accommodations. Disertación doctoral no publicada, University of Arkansas, Fayetteville.

ROSE, D. \& MEYER, A. (2002). Teaching every Student in the Digital Age: Universal Design for Learning. Alexandria, VA: ASCD.

ROWLAND, C. (2000). University-Affiliated Programs Face Web Site Accessibility Issues. CPD News, 22 (3), 1-5.

SÁNCHEZ HÍPOLA, P. (Dir.) (2008). Enseñanza accesible en la Universidad: guía didáctica para el profesorado. Madrid: Cátedra Microsoft de Accesibilidad a la Educación. http://ccae.mat.ucm.es/ccae/recursos/documentos/guias-deaccesibilidad/Guia_PIMCD08.pdf

STODEN, R. (2001). Postsecondary Education Supports for Students with Disabilities: A Review and Response. Journal for Vocational Special Needs Education, 23, 34-46.

TÉRMENS, M. \& RIBERA, M (2002). La accesibilidad de las universidades españolas en la web. Red de Integración Especial Tercer Congreso Virtual "Integración sin barreras en el siglo XXI".

TINCANI, M. (2004). Improving outcomes for College Students with Disabilities: Ten Strategies for Instructors. College Teaching, 52 (4), 128-132.

TOLEDO, P. (2001). La accesibilidad en las webs de las universidades andaluzas. Píxel- Bit, 17, 53-66.

VASEK, D. (2005). Asesing the Knowledge Base of Facult at a Private, Four-Year Institution College. Student Journal, Jun 2005.

WEST, M.; KREGER, J.; GETZEL, E.E.; ZHU M.; IPSEN, S. M. \& MARTIN, E.D. (1993). Beyond Section 504: Satisfaction and empowerment of students with disabilities in higher education. Exceptional Children, 59, 456-467.

WILLIAMSON, P.T. (2000). Attitudes of the Troy State University Dothan faculty towards students with disabilities. Nueva York: WorldCat OCLC.

ZEFF, R. (2007). Universal Design across the Curriculum. New Directions for Higher Education, 137, 27-44.

ZUBILLAGA, A. (2006). Enseñanza virtual accesible: Principios para el desarrollo de entornos virtuales de enseñanza y aprendizaje accesibles. En Rodríguez, J; Sánchez, R. \& Soto, J. Las Tecnologías en la Escuela Inclusiva: nuevos escenarios, nuevas oportunidades. Murcia: Consejería de Educación y Cultura. Región de Murcia y Ministerio de Trabajo y Asuntos Sociales. Real Patronato sobre Discapacidad. 
ZUBILLAGA DEL RÍO, A. (Dir.) (2008). Guía para una enseñanza virtual accesible. Madrid: Cátedra Microsoft de Accesibilidad a la Educación. http://ccae.mat.ucm.es/ccae/recursos/documentos/guiasdeaccesibilidad/guia-docente-para-una-ensenanza-virtual-accesible-1/indice

\section{Correspondencia con las autoras}

Carmen Alba Pastor

Facultad de Educación

Universidad Complutense de Madrid

Rector Royo Villanova s/n

28040 Madrid, España

E-mail: carmenal@edu.ucm.es

Ainara Zubillaga del Río

Universidad Camilo José Cela

Calle de Alarcón, 49

28692 Madrid

E-mail: azubillaga@ucjc.edu 